\title{
Técnica de Stoppa modificada para fratura de acetábulo: uma revisão*
}

\section{Modified Stoppa Approach for Acetabulum Fracture: A Review}

\author{
Ashwani Soni ${ }^{1}$ Ravi Gupta ${ }^{1}$ Ramesh Sen ${ }^{2}$ \\ ${ }^{1}$ Departmento de Ortopedia, Government Medical College and \\ Hospital, Chandigarh, India \\ ${ }^{2}$ Departmento de Ortopedia, Fortis Hospital Mohali, Sahibzada Ajit \\ Singh Nagar, India
}

\author{
Address for correspondence Ashwani Soni, Department of
Orthopedics, Government Medical College and Hospital, Chandigarh, \\ Address for correspondence Ashwani Soni, Department of
Orthopedics, Government Medical College and Hospital, Chandigarh, \\ India (e-mail: asoniortho@gmail.com). \\ India (e-mail asoniortho@gmailcom).
}

\section{Resumo \\ Palavras-chave \\ - acetábulo \\ - fraturas ósseas \\ - fixação de fratura \\ - Stoppa modificado}

Fraturas acetabulares são lesões graves e desafiadoras para cirurgiões ortopédicos. As abordagens anterior e posterior foram descritas para a fixação dessas fraturas. A técnica de Stoppa modificada é considerada uma alternativa à abordagem anterior, que fornece acesso à pelve pelo interior da cavidade. $O$ objetivo do presente artigo é revisar a literatura disponível sobre o uso da abordagem de Stoppa modificada em fraturas de acetábulo. Os dados disponíveis sugerem que a abordagem de Stoppa modificada é útil em fraturas anteriores de acetábulo e em algumas fraturas posteriores, especialmente aquelas nas quais o reforço da parede medial se faz necessário.

Fracture of the acetabulum is a grave injury and a challenge for orthopedic surgeons. Anterior and posterior approaches have been described for fixation of acetabulum fractures. The modified Stoppa approach is considered an alternate to the anterior approach, which provides access to the pelvis from inside the cavity. The purpose of the present article is to review the available literature on the use of the modified Stoppa approach in acetabulum fractures. The available data suggest that the modified Stoppa approach is useful in anterior acetabulum fractures and some posterior acetabulum fractures, especially those in which buttressing of the medial wall is required.

\section{Introdução}

O tratamento das fraturas do acetábulo é um desafio para um cirurgião ortopédico. $O$ entendimento da anatomia tridimensional e o reparo correto da fratura são essenciais para o tratamento. Após o trabalho pioneiro de Judet ${ }^{1}$ e Letournel, ${ }^{2}$ que descreveram o sistema de classificação e as abordagens, várias modificações foram desenvolvidas para melhorar 0 resultado. A abordagem ilioinguinal foi originalmente descrita

Estudo conduzido no Department of Orthopedics, Government Medical College and Hospital, Chandigarh, Índia.

received

August 5, 2017

accepted

September 28, 2018

published online

April 15, 2019 por Letournel ${ }^{3}$ em 1961 para a fixação do acetábulo fraturado. Como abordagem intrapélvica, tornou-se popular para a fixação de fraturas da parede e da coluna anterior, fraturas anteriores associadas a fraturas pós-hemitransversas e até mesmo ambas as fraturas da coluna.

A abordagem de Stoppa tem sido utilizada no tratamento de hérnias inguinais. ${ }^{4,5}$ Hirvensalo et $\mathrm{al}^{6}{ }^{6}$ em 1993, e Cole e Bolhofner, ${ }^{7}$ em 1994, foram os primeiros a independentemente descrever as abordagens extraperitoneais intrapélvicas para fixação de fraturas do acetábulo pélvico, uma modificação da abordagem de Stoppa.

Copyright $\odot 2019$ by Sociedade Brasileira License terms de Ortopedia e Traumatologia. Published by Thieme Revnter Publicações Ltda, Rio de Janeiro, Brazil

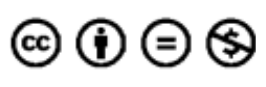

DOI https://doi.org/

10.1016/j.rboe.2017.09.006. ISSN 0102-3616. 
A abordagem de Stoppa modificada dá acesso direto aos ossos púbicos, à superfície posterior do ramo, à superfície quadrilátera, à eminência púbica e à superfície infrapectínea. Essa abordagem também dá acesso ao pilar ciático, incisura ciática e articulação sacroilíaca anterior. Essa abordagem é associada a uma vantagem mecânica na redução e fixação de fraturas de deslocamento medial em comparação a outras abordagens e é considerada uma alternativa viável de abordagem ilioinguinal para tratamento de fraturas do acetábulo anterior. Embora existam estudos sobre o uso da abordagem de Stoppa modificada em fraturas do acetábulo, não há artigos de revisão. Pela primeira vez, revisamos a literatura sobre a modificação da abordagem de Stoppa para fraturas do acetábulo.

\section{Materiais e Métodos}

A metodologia é descrita no fluxograma Preferred Reporting Items for Systematic Reviews and Meta-Analyses (PRISMA, na sigla em inglês) (-Fig. 1). Os bancos de dados Pubmed/ Medline e Cochrane foram pesquisados com as palavraschave Stoppa modificada, intra-pélvico anterior, intrapélvico anterior, Pfannensteil extenso, ílio anterior e terceira janela ilioinguinal (no original, em inglês, modified stoppa, anterior intra pelvic, anterior intrapelvic, extended pfannensteil, ilio anterior e third window ilioinguinal) para recuperação de artigos em que a abordagem de Stoppa foi usada na fixação de fraturas do acetábulo (em 22 de dezembro de 2016). As palavras-chave utilizadas foram os nomes alternativos que descrevem a abordagem de Stoppa modificada na literatura. Os critérios de inclusão foram todos os artigos em que a abordagem de Stoppa modificada foi usada na fixação de fraturas do acetábulo. Os critérios de exclusão foram artigos em língua não-inglesa, relatos de casos, estudos com cadáveres, artigos sem experiência clínica, lesões associadas do anel pélvico, e artigos em que a abordagem ilioinguinal foi utilizada. Os artigos foram avaliados quanto aos dados demográficos, modo de lesão, classificação da fratura, tempo até a cirurgia, tempo cirúrgico, perda de sangue, outras abordagens utilizadas, desfechos funcionais e radiológicos e complicações. No total, 552 artigos foram encontrados. Destes, 506 artigos não eram relacionados à abordagem de Stoppa modificada para fraturas do acetábulo. Nove artigos referentes à abordagem de Stoppa modificada para fraturas de acetábulo em idioma diferente do inglês foram excluídos. Três estudos com cadáveres ${ }^{8-10}$ e dois relatos de caso ${ }^{11,12}$ foram excluídos. Nove artigos ${ }^{13-21}$ foram excluídos porque a abordagem de Stoppa modificada foi descrita como uma técnica sem experiência clínica. Três artigos foram excluídos por incluírem o tratamento de pacientes com lesões do anel pélvico. ${ }^{22-24} \mathrm{Um}$ artigo de Chesser et $\mathrm{al}^{25}$ foi excluído por incluir pacientes tratados com a abordagem ilioinguinal. Um

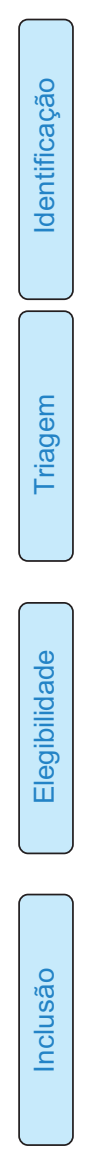

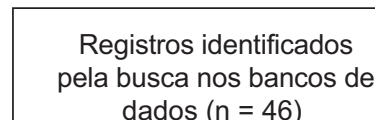
dados $(n=46)$

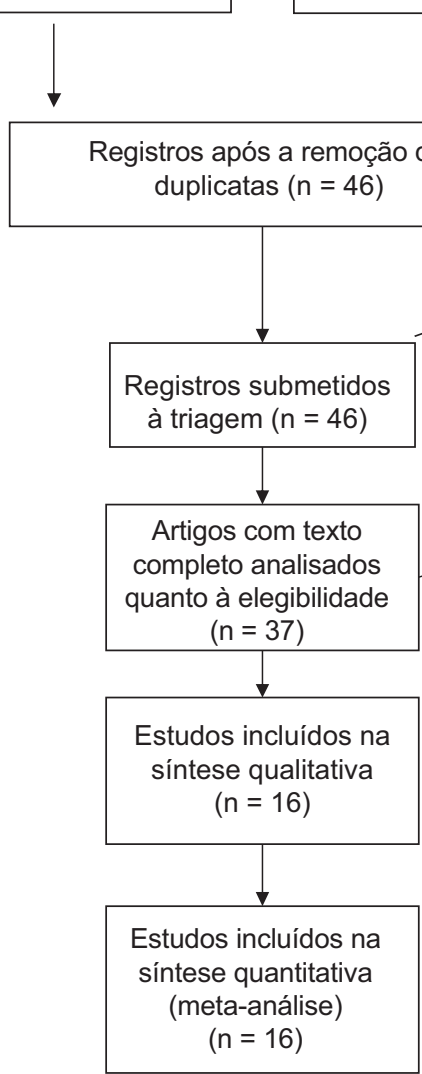

Outros registros

identificados por outras fontes $(n=0)$
Registros excluídos (idioma não inglês) $(n=9)$

Registros submetidos 7 $\rightarrow$ Artigos com texto completo excluídos, com justificativa (mencionados no texto) $(n=21)$

Fig. 1 Fluxograma PRISMA. 
artigo de Bastian et al $^{26}$ foi excluído porque a abordagem de Stoppa modificada foi alterada para a abordagem ilioinguinal no período intraoperatório em $9 \%$ dos pacientes, e os resultados das duas técnicas foram combinados. Dois artigos de Keel et $\mathrm{al}^{27,28}$ foram excluídos. Nestes estudos, o autor descreveu a abordagem pararretal, em que a fratura foi abordada de um plano lateral aos músculos retos. 0 próprio autor descreveu sua técnica de forma diferente da abordagem de Stoppa modificada e afirmou sua superioridade.

\section{Resultados}

No total, 16 artigos atendiam aos nossos critérios de seleção, inclusive um estudo retrospectivo de Rocca et al, ${ }^{29}$ que comparava a abordagem ilioinguinal à abordagem de Stoppa. Os dados demográficos dos pacientes foram descritos de maneira combinada para as duas abordagens, mas os resultados foram analisados separadamente. Incluímos este artigo em nossa revisão ao analisar os resultados (redução pós-operatória pelo método de Matta, desfecho funcional com a modificação de Matta da pontuação Merle d'Aubinne e critérios radiológicos de Matta), mas o excluímos da análise dos dados demográficos dos pacientes. As referências de todos os 16 artigos foram manualmente pesquisadas para detecção de qualquer artigo ausente. Os dados analisados foram idade, razão sexual, modo de lesão, classificação de fratura, tempo até a cirurgia, tempo cirúrgico, perda de sangue, outras abordagens utilizadas, desfechos funcionais e radiológicos e complicações.

\section{Dados Demográficos}

Em 16 estudos, 609 pacientes com fratura de acetábulo foram tratados com a abordagem de Stoppa modificada. ${ }^{7,30-43}$ Havia 427 homens e 139 mulheres. A proporção entre homens e mulheres era conhecida para 566 pacientes. ${ }^{7,30-43}$ Do total de 609 pacientes, os 34 pacientes tratados por Rocca et al $^{29}$ foram excluídos porque seus dados demográficos foram descritos combinados àqueles de pacientes submetidos à abordagem ilioinguinal. Dos 10 pacientes tratados por Casstevens et $a{ }^{36}{ }^{36} \mathrm{a}$ proporção de mulheres e homens era conhecida para 8 pacientes. Dos 57 pacientes tratados por Sagi et al, ${ }^{31} 7$ apresentavam esqueleto imaturo e foram avaliados apenas quanto à abordagem cirúrgica, tempo cirúrgico, perda de sangue e complicações. Todos os estudos apresentavam mais pacientes do sexo masculino do que feminino.

Em 11 estudos (326 pacientes), a idade variou entre $10 \mathrm{e}$ 88 anos. ${ }^{7,30-35,37,39,41,42}$ A idade foi descrita como média por Elmadag et al, ${ }^{40}$ Shazar et al $^{38}$ e Dailey et al, ${ }^{43}$ e as médias foram 49,3 anos, 41,88 $\pm 15,7$ anos e 55,88 $\pm 20,31$ anos, respectivamente.

\section{Modo de lesão}

O modo de lesão foi avaliado em 6 estudos (271 pacientes). ${ }^{31-33,35,39,42,43}$ Os acidentes de trânsito foram o modo mais comum em 129 pacientes, seguido de queda de altura em 108 pacientes, acidente industrial em 6 pacientes, lesão por esmagamento em 3 pacientes e lesão esportiva em um paciente. Cole et $\mathrm{al}^{7}$ relataram os acidentes de trânsito como o modo mais comum de lesão em 83\% dos seus 55 pacientes.

\section{Classificação das fraturas}

Em 14 estudos, 456 pacientes foram classificados de acordo com o método de Judet e Letournel. ${ }^{7,30-42}$ Houve 128 lesões nas duas colunas, 89 lesões na coluna anterior com acometimento hemitransversal posterior, 84 lesões na coluna anterior, 54 lesões de tipo T, 53 lesões transversais, 29 lesões transversais com acometimento da parede posterior, 13 lesões na coluna anterior, 3 lesões na coluna anterior com acometimento da parede, 2 fraturas da parede anterior e 1 lesão na coluna posterior. $O$ padrão de fratura mais comum foi a fratura das duas colunas, em seis estudos, e da coluna anterior, em cinco estudos. Dailey et $\mathrm{al}^{43}$ utilizaram a abordagem intrapélvica anterior (AIA) em pacientes com fraturas das duas colunas e da coluna anterior associada à fratura hemitransversal posterior apenas em 112 pacientes.

\section{Tempo até a cirurgia}

O tempo transcorrido até a cirurgia foi mencionado em 8 estudos (260 pacientes) e variou de 0 a 30 dias. ${ }^{7,31-35,39-41} \mathrm{O}$ tempo médio entre a lesão e a cirurgia foi de $4,83 \pm 3,74$ no estudo de Dailey et al. ${ }^{43}$

\section{Tempo cirúrgico}

O tempo cirúrgico variou de 80 minutos a 568 minutos em 7 estudos (250 pacientes). 7,30,33,35,39,41,42 Anderson et al $^{30}$ descreveram o tempo cirúrgico como o período até a desmontagem do campo estéril, a obtenção de radiografias pósoperatórias e a revisão destas imagens pelo médico assistente após a revelação, um processo que leva pelo menos 30 minutos. Em seu estudo, o tempo variou de 3 horas a 8 horas e 48 minutos. Casstevens et $\mathrm{al}^{36}$ descreveram o tempo cirúrgico como mediano, com 232 minutos para os 10 pacientes. Shazar et al, ${ }^{38}$ Laflamme et al $^{32}$ e Dailey et al. ${ }^{43}$ descreveram o tempo cirúrgico como média, com 240,5 minutos, $167 \pm 41$ minutos e $276,59 \pm 103,66$ para seus 103 pacientes, 21 pacientes e 112 pacientes, respectivamente.

\section{Perda de sangue}

A perda estimada de sangue em 9 estudos (299 pacientes) variou de $100 \mathrm{~mL}$ a $5.000 \mathrm{~mL} .^{7,29,30,32,33,35,39-41}$ Casstevens et $\mathrm{al}^{36}{ }^{36}$ Laflamme et $\mathrm{al}^{32}$ e Dailey et $\mathrm{al}^{43}$ descreveram a perda de sangue como média de $1.270 \mathrm{~mL}, 1.376 \pm 608 \mathrm{~mL}$ e $1.159,73 \pm 1.072,5 \mathrm{~mL}$ em 10 pacientes, 21 pacientes e 112 pacientes, respectivamente.

\section{Abordagem}

A abordagem de Stoppa modificada foi combinada com Kocher-Langenbeck (K-L), janela lateral e janela medial da abordagem ilioinguinal em vários estudos (-Tabela 1).

\section{Desfecho}

\section{Redução pós-operatória - Método de Matta}

A redução pós-operatória da fratura foi avaliada em 12 estudos (408 pacientes) com o sistema de pontuação descrito por Matta ${ }^{44}$ em 1996. ${ }^{30-35,37-39,41,42}$ A redução foi anatômica ( $\leq 1 \mathrm{~mm}$ ) em 290 pacientes, imperfeita $(>1 \mathrm{a}<3 \mathrm{~mm}$ ) em 77 pacientes e má ( $\geq 3 \mathrm{~mm}$ ) em 34 pacientes. Sete 
112 Técnica de Stoppa modificada para fratura de acetábulo Soni et al.

Tabela 1 Abordagens usadas pelos autores

\begin{tabular}{|c|c|c|c|}
\hline Número & Autores & Número de pacientes & Abordagem \\
\hline 1 & Elmadağ et al $\left.\right|^{33}$ & 36 & MS \\
\hline 2 & Hammad et al ${ }^{42}$ & 21 & $\begin{array}{l}\mathrm{MS}+/ \text { - LW } \\
14 \% \text { requerem K-L }\end{array}$ \\
\hline 3 & Kim et $\mathrm{al}^{34}$ & 22 & $\begin{array}{l}\text { MS - } 13 \\
M S+K-L-9(5 \text { ACPHT, } 4 \text { Trans + PW) } \\
M S+L W-2\end{array}$ \\
\hline 4 & Rocca et al ${ }^{29}$ & 34 & MS + LW (abordagem ECA) \\
\hline 5 & Elmadağ et al ${ }^{40}$ & 17 & MS \\
\hline 6 & Shazar et al ${ }^{38}$ & 103 & $\begin{array}{l}\mathrm{MS}-32 \\
\mathrm{MS}+\mathrm{LW}-57 \\
\mathrm{MS}+\mathrm{K}-\mathrm{L}-11 \\
\mathrm{MS}+\mathrm{MW}+\mathrm{LW}-2 \\
\mathrm{MS}+\mathrm{SP}-1\end{array}$ \\
\hline 7 & Laflamme et al ${ }^{37}$ & 9 & $\mathrm{MS}+\mathrm{LW}$ \\
\hline 8 & Casstevens et al ${ }^{36}$ & 10 & MS \\
\hline 9 & Liu et $\mathrm{al}^{35}$ & 29 & MS + LW (nova abordagem de Stoppa modificada) \\
\hline \multirow[t]{2}{*}{10} & Ma et al ${ }^{39}$ & 30 & MS -8 \\
\hline & & & $\mathrm{MS}+\mathrm{LW}-22$ \\
\hline 11 & Andersen et al ${ }^{30}$ & 17 & $\begin{array}{l}\text { MS + Pós abordagem - excluído do estudo } \\
\text { MS + LW - incluído } \\
\text { Número exato não mencionado }\end{array}$ \\
\hline 12 & Sagi et $\mathrm{al}^{31}$ & $\begin{array}{l}57 \\
\text { (7 esqueletos imaturos) }\end{array}$ & $\begin{array}{l}\text { MS - } 23 \\
\text { MS }+ \text { LW - } 34 \text { (mais SM em } 2 \text { pacientes) (abordagem AIP) }\end{array}$ \\
\hline 13 & Isaacson et al ${ }^{41}$ & 36 & $\begin{array}{l}\mathrm{MS}+\mathrm{LW}-23 \\
\mathrm{MS}+\mathrm{K}-\mathrm{L}-3\end{array}$ \\
\hline 14 & Laflamme et al ${ }^{32}$ & 21 & $\mathrm{MS}+\mathrm{LW}$ \\
\hline 15 & Cole et $\mathrm{al}^{7}$ & 55 & $\begin{array}{l}\text { Apenas MS }-32 \\
\text { MS }+ \text { KL }-18 \\
\text { ( } 6 \text { pacientes precisaram da abordagem Avila lateral além da } \\
\text { abordagem principalmente anterior) }\end{array}$ \\
\hline 16 & Dailey et a $\left.\right|^{43}$ & 112 & Abordagem AIP (descrita por Sagi et al ${ }^{9}$ ) \\
\hline
\end{tabular}

Abreviaturas: ACPHT, Fratura hemitransversal posterior da coluna anterior; K-L, Kocher-Langenbeck; LW, Janela lateral do ilioinguinal; MS, Stoppa modificado; MW, Janela medial do ilioinguinal; SP, Smith Peterson; Trans + PW, Transversal com fratura da parede posterior.

pacientes apresentaram reduções anatômicas ou imperfeitas. Hammad et $\mathrm{al}^{42}$ verificaram que, dos 10 pacientes com desfecho desfavorável, 6 apresentavam acetábulo tipo B e que, dentre os 10 pacientes com redução anatômica, 6 apresentavam fratura tipo $C$. Sagi et $\mathrm{al}^{31}$ constataram que as más reduções de fratura ocorreram com maior frequência (75\%) em fraturas associadas nas duas colunas. Em seus resultados, a porcentagem de redução excelente foi máxima (92\%) nas fraturas da coluna anterior, enquanto a porcentagem de redução excelente foi mínima (67\%) nas fraturas transversais. Da mesma forma, o menor percentual de redução excelente (75\%) foi observado nas fraturas transversais por Shazar et al $^{38}$ mas, em seu estudo, a maior porcentagem $(92,9 \%)$ de redução anatômica foi alcançada na coluna anterior com fraturas hemitransversais posteriores.

\section{Pontuação do Quadril de Harris}

A pontuação do quadril de Harris foi avaliada em quatro estudos. $^{32,35,37,40} \mathrm{Em} 3$ estudos (82 pacientes), a pontuação foi excelente em 35, boa em 34, moderada em 8 e má em $5 .^{32,35,40}$ Laflamme et al $^{37}$ relataram uma pontuação média de 81(entre 51 e 100) em uma série de 9 pacientes.

\section{Pontuação de Merle d'Aubinne}

Em 4 estudos ${ }^{30,32,39,40}$ (133 pacientes), a pontuação de Merle d'Aubinne $^{45,46}$ foi excelente em 58 casos, boa em 59, moderada em 8 e má em 8 . No estudo de Isaacson et al, ${ }^{41}$ a pontuação de Merle d'Aubinne foi muito boa em 12 casos, boa em dois, média em 4 , moderada em 1 e má em $3 .^{45}$

\section{Modificação de Matta da pontuação de Merle d'Aubinne}

A modificação de Matta da pontuação de Merle d'Aubinne $^{44,47}$ foi utilizada para avaliação dos resultados funcionais de 3 estudos ${ }^{33,34,42}$ (72 pacientes). Quinze pacientes apresentaram resultados excelentes, 30 apresentaram bons resultados, 11 , moderados e 16 , maus. Cole et $\mathrm{al}^{7}$ descreveram os resultados funcionais de acordo com a modificação de 
Matta da pontuação de Merle d'Aubinne ${ }^{47}$ em porcentagem. Os autores obtiveram $47 \%$ de resultados excelentes, $42 \%$ bons, $9 \%$ moderados e $2 \%$ maus.

\section{Critérios radiológicos de Matta}

Em 3 estudos ${ }^{34,36,39}$ (70 pacientes), os resultados radiológicos foram classificados de acordo com Matta. ${ }^{44,47}$ Quarenta e cinco resultados excelentes, 14 bons, 6 moderados e 5 maus foram observados. Cole et $\mathrm{al}^{7}$ descreveram a pontuação radiológica de acordo com os critérios radiológicos de Matta $^{47}$ em porcentagem. Os autores obtiveram $64 \%$ de resultados excelentes, $25 \%$ bons, $7 \%$ moderados e $4 \%$ maus. Laflamme et $\mathrm{al}^{32}$ avaliaram os resultados funcionais da fixação interna das fraturas acetabulares osteopênicas com acometimento da placa quadrilátera em 19 pacientes idosos. ${ }^{31}$ Estes autores observaram (média $+\mid$ - desvio-padrão) SF-12 PCS - 45,3 ( $\pm 12,1)$, SF-12 MCS - 55,9 ( $\pm 8,5)$, PMA $16,8( \pm 1,3)$, HHS (pontuação do quadril de Harris) - 86,2 $( \pm 13,9)$, WOMAC (dor) - 3,4 ( $\pm 3,5)$, WOMAC (rigidez) - 1,3 $( \pm 1,5)$, WOMAC (ADL) - 11,7 ( $\pm 12,2)$.

\section{Complicações}

A lesão do nervo obturador foi a complicação mais frequente, e a artrite da articulação do quadril foi a complicação tardia mais comum (-Tabela $\mathbf{2}$ ).

\section{Discussão}

Judet et $\mathrm{al}^{48}$ descreveram o acetábulo como um alvéolo circundado e sustentado por um arco formado por duas colunas de ossos, anterior e posterior, e o teto do acetábulo como a peça principal desse arco. Estes autores enfatizaram a importância dessas colunas, salientando que seu restauro é necessário à correção de fraturas do acetábulo.

As abordagens clássicas para a fixação da fratura da coluna anterior e posterior são, respectivamente, K-L e ilioinguinal. No entanto, com o passar do tempo, outras abordagens para o acetábulo anterior foram desenvolvidas.

Hirvensalo et al, ${ }^{6}$ em 1993, e Cole et al, ${ }^{7}$ em 1994, foram os primeiros a descrever o uso do espaço pré-peritoneal no reparo de fraturas pélvicas. Hirvensalo et $\mathrm{al}^{6}$ trataram 18 lesões no anel pélvico com a abordagem baixa de Pfannensteil. Os músculos reto do abdômen e pectíneo foram separados dos ramos e a dissecção subperióstea foi realizada pela superfície interna da borda pélvica até a articulação sacroilíaca. A fáscia iliopectínea, os vasos ilíacos externos, o nervo femoral e o músculo psoas foram poupados. Os vasos e nervos obturadores, além da corona mortis, foram protegidos. A abordagem foi denominada "abordagem ilioanterior". A janela lateral da abordagem ilioinguinal foi usada com esta abordagem ilioanterior quando a fratura se estendia àquela região. Como este estudo incluía lesões do anel pélvico, ele foi removido de nossa revisão. Cole e Bolhofner ${ }^{7}$ trataram 55 fraturas acetabulares com a abordagem intrapélvica, que denominaram "Pfennensteil Estendida”, mas que, após maior discussão e pesquisa, passou a ser chamada abordagem "de Stoppa modificada", bastante similar à abordagem de Stoppa utilizada no reparo de hérnias. Os autores estenderam a abordagem de Stoppa posteriormente ao longo da borda, elevando o iliopectíneo e a fáscia obturadora. Os autores concluíram que essa nova abordagem pode melhorar a redução e a fixação de certos tipos de fraturas do acetábulo e diminuir a taxa de complicações associadas às abordagens com extensão extrapélvica.

\section{Alternativa à abordagem ilioinguinal}

A abordagem de Stoppa modificada, associada ou não a outras abordagens, é uma alternativa à abordagem ilioinguinal para a fixação das fraturas do acetábulo anterior. ${ }^{30,32-35,39}$ Elmadag et al $^{33}$ concluíram em seu estudo retrospectivo de 36 pacientes que a abordagem de Stoppa modificada era uma boa alternativa à abordagem ilioinguinal mesmo em muitas fraturas acetabulares complexas, apesar da acentuada curva de aprendizado devido à proximidade de estruturas neurovasculares. Kim et al, ${ }^{34}$ em seu estudo com 22 pacientes, concluíram que a abordagem de Stoppa modificada pode ser uma alternativa à abordagem ilioinguinal clássica, com resultados excelentes e bons; entretanto, a fragmentação da fratura acetabular é um fator importante, levando à redução não anatômica e resultados clínicos insatisfatórios.

\section{Acesso à placa quadrilátera}

A abordagem de Stoppa modificada permite o acesso ao acetábulo por dentro da pelve, facilitando a abordagem das fraturas com acometimento da placa quadrilátera. ${ }^{30,31,34,35,42}$ Laflamme et al $^{32}$ afirmaram que a placa quadrangular pode ser fixada tão bem com essa abordagem que pode ser uma alternativa viável à artroplastia em pacientes idosos ou com osteoporose e determinadas fraturas acetabulares. Os autores trataram 21 pacientes com mais de 60 anos de idade ou osteoporose estabelecida e fraturas do acetábulo com acometimento da placa quadrilátera. A abordagem de Stoppa modificada, juntamente com a janela lateral, foi usada na fixação de fraturas do acetábulo e fortalecimento da placa quadrilátera. Os autores concluíram que o tratamento inicial da fratura acetabular osteoporótica envolvendo a coluna anterior e a placa quadrilátera com fixação pela abordagem de Stoppa modificada para sustentação da placa quadrilátera deve ser considerado como alternativa viável à artroplastia total do quadril.

\section{Acesso à coluna posterior}

A abordagem de Stoppa modificada combinada à janela lateral da abordagem ilioinguinal também permite o acesso à coluna posterior. Isso ajuda a fixação da fratura do acetábulo anterior que se estende até o acetábulo posterior. ${ }^{29,30,41}$ Usando a abordagem de Stoppa modificada juntamente com a janela lateral da abordagem ilioinguinal, Andersen et al trataram 17 pacientes jovens (idade $<60$ anos) com fraturas do acetábulo e deslocamento da coluna anterior e posterior. ${ }^{29}$ Os autores concluíram que, em certas fraturas com deslocamento das colunas anterior e posterior (mesmo com mais de $5 \mathrm{~mm}$ de deslocamento da coluna posterior), a redução anatômica ou imperfeita pode ser obtida com a abordagem de Stoppa modificada e a janela lateral da abordagem ilioinguinal. Isaacson et al, em seu estudo prospectivo com 36 pacientes, relataram bom resultado funcional com 
114 Técnica de Stoppa modificada para fratura de acetábulo Soni et al.

Tabela 2 Complicações

\begin{tabular}{|c|c|c|}
\hline Complicações & $\begin{array}{l}\text { Número de } \\
\text { pacientes }\end{array}$ & Tratamento \\
\hline \multicolumn{3}{|l|}{ Complicações intraoperatórias } \\
\hline Lesão da corona mortis & 2 & $\begin{array}{l}\text { Hemostasia e ligadura em } 1 \text { paciente } \\
\text { Detalhes do paciente não foram mencionados }\end{array}$ \\
\hline Lesão da artéria obturadora & 1 & Hemostasia e ligadura \\
\hline Lesão da veia ilíaca externa & 1 & Reparo primário \\
\hline Lesão da artéria glútea superior & 1 & Hemostasia e embolização \\
\hline \multicolumn{3}{|l|}{ Complicações pós-operatórias } \\
\hline Lesão do nervo obturador & 21 & $\begin{array}{l}\text { Recuperação em 3-6 meses em } 20 \text { pacientes } \\
\text { Recuperação parcial em } 1 \text { paciente }\end{array}$ \\
\hline Infecção profunda & 13 & $\begin{array}{l}\text { Debridamento e antibióticos em } 11 \text { pacientes } \\
\text { Debridamento, antibióticos e remoção de placa em } 1 \text { paciente } \\
\text { Artroplastia do quadril em } 1 \text { paciente }\end{array}$ \\
\hline $\begin{array}{l}\text { Paralisia do nervo cutâneo } \\
\text { femoral lateral }\end{array}$ & 8 & $\begin{array}{l}\text { Recuperação em 3-6 meses em } 5 \text { pacientes } \\
\text { Detalhes de } 2 \text { pacientes não foram mencionados }\end{array}$ \\
\hline Pé caído (foot drop) & 5 & Recuperação em 6-12 meses \\
\hline Infecção superficial & 5 & Curativo e antibióticos \\
\hline Trombose venosa profunda & 7 & $\begin{array}{l}\text { Profilaxia química em } 1 \text { paciente } \\
\text { Detalhes de } 3 \text { pacientes não foram mencionados }\end{array}$ \\
\hline Parafuso intra-articular & 2 & Removido \\
\hline Paralisia do nervo ciático & 1 & Recuperação em 6 semanas \\
\hline Seroma no sítio cirúrgico & 1 & Tratamento cirúrgico, sem detecção de infecção \\
\hline Ruptura peritoneal & 1 & A ferida foi fechada sem sequelas \\
\hline Deiscência da ferida & 1 & O fechamento cirúrgico foi realizado \\
\hline Retardo da cicatrização & 1 & Cicatrização em 3 semanas, tratamento c/ infravermelho \\
\hline \multicolumn{3}{|l|}{ Complicações tardias } \\
\hline Artrite da articulação do quadril & 15 & \\
\hline Formação óssea ectópica & 9 & $\begin{array}{l}\text { Tratamento conservativo em } 3 \text { pacientes com amplitude } \\
\text { total de movimento } \\
\text { Detalhes de } 6 \text { pacientes não foram mencionados }\end{array}$ \\
\hline Necrose avascular da cabeça do fêmur & 5 & \\
\hline Perda de redução & 4 & $\begin{array}{l}\text { Artroplastia de quadril em } 2 \text { pacientes } \\
\text { Detalhes de } 2 \text { pacientes não foram mencionados }\end{array}$ \\
\hline Atrofia do reto sem hérnia & 2 & \\
\hline Hérnia inguinal lateral & 4 & Reparo realizado \\
\hline Não união & 1 & Fixação e enxerto ósseo em 10 meses \\
\hline \multicolumn{3}{|l|}{ Outras complicações } \\
\hline Embolia pulmonar fatal & 2 & \\
\hline Embolia pulmonar não fatal & 1 & \\
\hline Acidente vascular cerebral & 1 & \\
\hline Trombose da artéria ilíaca comum & 1 & \\
\hline Cirrose hepática fatal & 1 & \\
\hline
\end{tabular}

taxas mínimas de complicações com a técnica de Stoppa modificada em fraturas do acetábulo, inclusive fraturas com extensão e deslocamento da coluna posterior. ${ }^{41}$ No entanto, estes autores destacaram a dificuldade, mas a possibilidade, de tratamento do deslocamento da coluna posterior somente pela abordagem anterior.

\section{Variações da abordagem}

Embora muitos autores combinem a abordagem de Stoppa modificada com outras técnicas ( $\mathbf{- T a b e l a ~ 2 )}$ ), certas modificações também foram descritas. ${ }^{30,34,35}$ Rocca et al ${ }^{29}$ combinaram a abordagem de Stoppa modificada, descrita por Cole e Bolhofner, com as janelas proximal e lateral da abordagem 
ilioinguinal e a denominaram abordagem Endopélvica Combinada Anterior (ECA). Sagi et $\mathrm{al}^{31}$ descreveram a modificação da abordagem de Rives-Stoppa como abordagem intrapélvica anterior (AIP). A janela lateral da abordagem ilioinguinal foi usada, se necessário. A visualização excelente e o acesso à placa quadrilátera e à coluna posterior foram relatados. A redução foi boa a excelente na maioria dos casos, com taxas de complicações comparáveis às da abordagem ilioinguinal. Os autores consideraram a abordagem útil em caso de fragmentação da placa quadrilátera e coluna posterior e recomendaram esta técnica como uma possível alternativa à abordagem ilioinguinal para exposição anterior do acetábulo. Ao invés de usar a incisão na linha média, Liu et $\mathrm{al}^{35}$ trataram 29 fraturas do acetábulo através dos dois terços internos de uma linha $2 \mathrm{~cm}$ acima do ligamento inguinal e paralela a ele. A fáscia do reto foi dividida, a artéria epigástrica inferior foi ligada e a fáscia transversal foi incisada logo acima da sínfise púbica. Esta abordagem anterior foi combinada à abordagem lateral na crista ilíaca. A abordagem foi denominada "nova abordagem de Stoppa modificada". Os autores concluíram que esta abordagem proporciona excelente acesso à coluna anterior, à superfície quadrilátera e à incisura ciática, permite excelente redução e fixação, tem bons resultados pós-operatórios e é uma alternativa viável à abordagem ilioinguinal. As taxas de complicações foram consideradas comparáveis às da abordagem ilioinguinal.

\section{Diferentes artifícios técnicos}

Como o cirurgião pode abordar o acetábulo por dentro da pelve pela modificação da abordagem de Stoppa, diferentes artifícios técnicos foram descritos utilizando essa vantagem. Casstevens et al, ${ }^{36} \mathrm{em} 10$ pacientes, descreveram um artifício técnico usando a abordagem de Stoppa modificada para redução e fortalecimento da fixação do parafuso na cúpula impactada do acetábulo. Da mesma forma, Laflamme et al, ${ }^{37}$ em nove pacientes, descreveram o artifício técnico de redução direta da impactação da cúpula superomedial em fraturas acetabulares geriátricas usando a abordagem de Stoppa modificada.

\section{Tempo de cirurgia}

Encontramos apenas um estudo que avaliou o efeito do tempo cirúrgico da abordagem ilioinguinal modificada. Dailey et $\mathrm{al}^{43}$ avaliaram a influência do momento da cirurgia na perda de sangue e no tempo de cirurgia em pacientes com fratura do acetábulo. Foram tratados 176 pacientes com fratura da parede posterior do acetábulo com a abordagem de K-L. Um total de 112 pacientes com fratura das 2 colunas ou da coluna anterior e hemitransversal posterior foram tratados com a abordagem intrapélvica anterior. Nosso estudo incluiu 112 pacientes submetidos à abordagem intrapélvica anterior. $\mathrm{O}$ autor não encontrou diferença em termos de perda de sangue ou tempo cirúrgico nos casos de fixação precoce $(<48$ horas) ou tardia ( $>48$ horas) das fraturas, sejam na parede posterior ou associadas, em ambas as colunas/coluna anterior e hemitransversal posterior.

\section{Comparação com a abordagem ilioinguinal}

Cinco estudos compararam os resultados da abordagem de Stoppa e da abordagem ilioinguinal na fixação da fratura de acetábulo. $^{34,38-40,42}$ Rocca et al $^{29}$ trataram 34 pacientes com fratura de acetábulo com a abordagem ECA (abordagem de Stoppa modificada combinada à janela lateral de abordagem ilioinguinal) e os compararam com 42 pacientes submetidos à abordagem ilioinguinal. A técnica ECA foi considerada uma alternativa mais fácil e viável à abordagem ilioinguinal para exposição do acetábulo anterior e da placa quadrilátera com resultado clínico um pouco melhor e taxas similares de complicações. Hammad et al concluíram que a precisão da redução e as pontuações clínicas foram similares nas duas abordagens. ${ }^{42} \mathrm{~A}$ abordagem de Stoppa pode ser mais vantajosa no reforço direto da placa quadrilátera nas fraturas de ambas as colunas, enquanto a janela média da abordagem ilioinguinal expõe melhor as fraturas da parede anterior e em orientação transversal. Elmadağ et al ${ }^{40}$ concluíram que não há vantagem da abordagem de Stoppa em relação à abordagem ilioinguinal em termos de complicações e sangramento, exceto que a primeira gera cicatrizes de aspecto cosmético melhor. Além disso, a abordagem de Stoppa permite o tratamento de fraturas bilaterais com incisão única e melhora a visualização das lesões de compressão lateral. Shazar et a ${ }^{38}$ concluíram que a abordagem AIP é associada à maior taxa de redução anatômica em comparação à abordagem ilioinguinal e tem menor tempo cirúrgico e taxas de complicações comparáveis. Ma et $\mathrm{al}^{39}$ concluíram que não houve diferença significativa na qualidade da redução, nos resultados do acompanhamento por imagem, nos desfechos clínicos e nas complicações entre a abordagem de Stoppa modificada e a abordagem ilioinguinal na fixação da fratura do acetábulo. No entanto, o tempo cirúrgico, a perda de sangue, a drenagem das feridas e a transfusão de sangue são menores com a abordagem de Stoppa. Os autores recomendaram a abordagem de Stoppa para exposição do acetábulo anterior, em especial em fraturas com acometimento da placa quadrilátera.

Os estudos atuais sobre a abordagem de Stoppa modificada para o tratamento de fraturas do acetábulo são altamente variáveis em termos de delineamento experimental. Diferentes métodos de avaliação dos resultados foram utilizados por diferentes autores. Diferentes modificações e artifícios técnicos desta abordagem também foram descritos com diferentes objetivos e metas. Além disso, as fraturas associadas do anel pélvico e o uso adicional de outras abordagens para as fraturas do acetábulo dificultam a avaliação dos resultados da abordagem de Stoppa modificada nestas lesões.

\section{Conclusão}

Embora haja alta variabilidade de delineamentos experimentais em estudos anteriores, a literatura disponível sugere que a abordagem de Stoppa modificada é uma abordagem anterior útil na fixação de fraturas do acetábulo, especialmente para reforço da placa quadrilátera. As fraturas da coluna posterior podem ser abordadas por esta técnica ou corrigidas com a abordagem combinada de K-L. Nas fraturas que se 
estendem até a crista ilíaca, uma janela lateral pode ser feita sem expor o feixe neurovascular, como na abordagem ilioinguinal, que gera boa redução e taxas aceitáveis de complicações.

\section{Conflitos de Interesse}

Os autores declaram não haver conflitos de interesse.

\section{Referências}

1 Judet R, Judet J, Letournel E. [Surgical treatment of recent fractures of the acetabulum. (Apropos of 46 operated cases)]. Mem Acad Chir (Paris) 1962;88:369-377

2 Letournel E, Judet R. Fractures of the acetabulum. 2nd. New York: Springer-Verlag; 1974

3 Letournel E. Fractures of the cotyloid cavity, study of a series of 75 cases]. J Chir (Paris) 1961;82:47-87

4 Rives J, Stoppa R, Fortesa L, Nicaise H. [Dacron patches and their place in surgery of groin hernia. 65 cases collected from a complete series of 274 hernia operations]. Ann Chir 1968;22 (03):159-171

5 Stoppa RE, Rives JL, Warlaumont CR, Palot JP, Verhaeghe PJ, Delattre JF. The use of Dacron in the repair of hernias of the groin. Surg Clin North Am 1984;64(02):269-285

6 Hirvensalo E, Lindahl J, Böstman O. A new approach to the internal fixation of unstable pelvic fractures. Clin Orthop Relat Res 1993; (297):28-32

7 Cole JD, Bolhofner BR. Acetabular fracture fixation via a modified Stoppa limited intrapelvic approach. Description of operative technique and preliminary treatment results. Clin Orthop Relat Res 1994;(305):112-123

8 Bastian JD, Savic M, Cullmann JL, Zech WD, Djonov V, Keel MJ. Surgical exposures and options for instrumentation in acetabular fracture fixation: Pararectus approach versus the modified Stoppa. Injury 2016;47(03):695-701

9 Bible JE, Choxi AA, Kadakia RJ, Evans JM, Mir HR. Quantification of bony pelvic exposure through the modified Stoppa approach. J Orthop Trauma 2014;28(06):320-323

10 Kacra BK, Arazi M, Cicekcibasi AE, Büyükmumcu M, Demirci S. Modified medial Stoppa approach for acetabular fractures: an anatomic study. J Trauma 2011;71(05):1340-1344

11 Murcia-Asensio A, Ferrero-Manzanal F, Lax-Pérez R, FernándezFairén M. Stoppa approach for intrapelvic damage control and reconstruction of complex acetabular defects with intra-pelvic socket migration: A case report. Int J Surg Case Rep 2016;25:143-148

12 Elmadag M, Acar MA. A modified stoppa (technique) approach for treatment of pediatric acetabular fractures. Case Rep Orthop 2013;2013:478131

13 Archdeacon MT, Kazemi N, Guy P, Sagi HC. The modified Stoppa approach for acetabular fracture. J Am Acad Orthop Surg 2011;19 (03):170-175

14 Archdeacon MT. Comparison of the ilioinguinal approach and the anterior intrapelvic approaches for open reduction and internal fixation of the acetabulum.J Orthop Trauma 2015;29(Suppl 2):S6-S9

15 Collinge CA, Lebus GF. Techniques for reduction of the quadrilateral surface and dome impaction when using the anterior intrapelvic (modified Stoppa) approach. J Orthop Trauma 2015; 29(Suppl 2):S20-S24

16 Guy P. Evolution of the anterior intrapelvic (Stoppa) approach for acetabular fracture surgery. J Orthop Trauma 2015;29(Suppl 2):S1-S5

17 Hagen JE, Weatherford BM, Nascone JW, Sciadini MF. Anterior intrapelvic modification to the ilioinguinal approach. J Orthop Trauma 2015;29(Suppl 2):S10-S13

18 Khoury A, Weill Y, Mosheiff R. The Stoppa approach for acetabular fracture. Oper Orthop Traumatol 2012;24(4-5):439-448
19 Kistler BJ, Sagi HC. Reduction of the posterior column in displaced acetabulum fractures through the anterior intrapelvic approach. J Orthop Trauma 2015;29(Suppl 2):S14-S19

20 Qureshi AA, Archdeacon MT, Jenkins MA, Infante A, DiPasquale T, Bolhofner BR. Infrapectineal plating for acetabular fractures: a technical adjunct to internal fixation. J Orthop Trauma 2004;18 (03):175-178

21 Sagi HC, Bolhofner B. Osteotomy of the Anterior Superior Iliac Spine as an Adjunct to Improve Access and Visualization Through the Lateral Window. J Orthop Trauma 2015;29(08):e266-e269

22 Vikmanis A, Vikmanis A, Jakusonoka R, Jumtins A, Pavare Z. Midterm outcome of patients with pelvic and acetabular fractures following internal fixation through a modified Stoppa approach. Acta Orthop Belg 2013;79(06):660-666

23 Jakob M, Droeser R, Zobrist R, Messmer P, Regazzoni P. A less invasive anterior intrapelvic approach for the treatment of acetabular fractures and pelvic ring injuries. J Trauma 2006;60(06): $1364-1370$

24 Ponsen KJ, Joosse P, Schigt A, Goslings JC, Luitse JS. Internal fracturefixation using the Stoppa approach in pelvic ring and acetabular fractures: technical aspects and operative results. J Trauma 2006;61(03):662-667. Erratum in. J Trauma 2007;62 (6): 1490

25 Chesser TJ, Eardley W, Mattin A, Lindh AM, Acharya M, Ward AJ. The modified ilioinguinal and anterior intrapelvic approaches for acetabular fracture fixation: indications, quality of reduction, and early outcome. J Orthop Trauma 2015;29(Suppl 2):S25-S28

26 Bastian JD, Tannast M, Siebenrock KA, Keel MJ. Mid-term results in relation to age and analysis of predictive factors after fixation of acetabular fractures using the modified Stoppa approach. Injury 2013;44(12):1793-1798

27 Keel MJ, Tomagra S, Bonel HM, Siebenrock KA, Bastian JD. Clinical results of acetabular fracture management with the Pararectus approach. Injury 2014;45(12):1900-1907

28 Keel MJ, Ecker TM, Cullmann JL, Bergmann M, Bonel HM, Büchler $\mathrm{L}$, et al. The Pararectus approach for anterior intrapelvic management of acetabular fractures: an anatomical study and clinical evaluation. J Bone Joint Surg Br 2012;94(03):405-411

29 Rocca G, Spina M, Mazzi M. Anterior Combined Endopelvic (ACE) approach for the treatment of acetabular and pelvic ring fractures: A new proposal. Injury 2014;45(Suppl 6):S9-S15

30 Andersen RC, O'Toole RV, Nascone JW, Sciadini MF, Frisch HM, Turen CW. Modified stoppa approach for acetabular fractures with anterior and posterior column displacement: quantification of radiographic reduction and analysis of interobserver variability. J Orthop Trauma 2010;24(05):271-278

31 Sagi HC, Afsari A, Dziadosz D. The anterior intra-pelvic (modified rives-stoppa) approach for fixation of acetabular fractures. J Orthop Trauma 2010;24(05):263-270

32 Laflamme GY, Hebert-Davies J, Rouleau D, Benoit B, Leduc S. Internal fixation of osteopenic acetabular fractures involving the quadrilateral plate. Injury 2011;42(10):1130-1134

33 Elmadag M, Guzel Y, Aksoy Y, Arazi M. Surgical Treatment of Displaced Acetabular Fractures Using a Modified Stoppa Approach. Orthopedics 2016;39(02):e340-e345

34 Kim HY, Yang DS, Park CK, Choy WS. Modified Stoppa approach for surgical treatment of acetabular fracture. Clin Orthop Surg 2015;7 (01):29-38

35 Liu Y, Yang H, Li X, Yang SH, Lin JH. Newly modified Stoppa approach for acetabular fractures. Int Orthop 2013;37(07): 1347-1353

36 Casstevens C, Archdeacon MT, d'Heurle A, Finnan R. Intrapelvic reduction and buttress screw stabilization of dome impaction of the acetabulum: a technical trick. J Orthop Trauma 2014;28(06): e133-e137

37 Laflamme GY, Hebert-Davies J. Direct reduction technique for superomedial dome impaction in geriatric acetabular fractures. J Orthop Trauma 2014;28(02):e39-e43 
38 Shazar N, Eshed I, Ackshota N, Hershkovich O, Khazanov A, Herman A. Comparison of acetabular fracture reduction quality by the ilioinguinal or the anterior intrapelvic (modified RivesStoppa) surgical approaches. J Orthop Trauma 2014;28(06): 313-319

39 Ma K, Luan F, Wang X, Ao Y, Liang Y, Fang Y, et al. Randomized, controlled trial of the modified Stoppa versus the ilioinguinal approach for acetabular fractures. Orthopedics 2013;36(10): e1307-e1315

40 Elmadağ M, Güzel Y, Acar MA, Uzer G, Arazi M. The Stoppa approach versus the ilioinguinal approach for anterior acetabular fractures: a case control study assessing blood loss complications and function outcomes. Orthop Traumatol Surg Res 2014;100 (06):675-680

41 Isaacson MJ, Taylor BC, French BG, Poka A. Treatment of acetabulum fractures through the modified Stoppa approach: strategies and outcomes. Clin Orthop Relat Res 2014;472(11): 3345-3352

42 Hammad AS, El-Khadrawe TA. Accuracy of reduction and early clinical outcome in acetabular fractures treated by the standard ilio-inguinal versus the Stoppa/iliac approaches. Injury 2015;46 (02):320-326

43 Dailey SK, Archdeacon MT. Open reduction and internal fixation of acetabulum fractures: does timing of surgery affect blood loss and OR time? J Orthop Trauma 2014;28(09):497-501

44 Matta JM. Fractures of the acetabulum: accuracy of reduction and clinical results in patients managed operatively within three weeks after the injury. J Bone Joint Surg Am 1996;78(11): 1632-1645

45 D'Aubigne RM, Postel M. Functional results of hip arthroplasty with acrylic prosthesis. J Bone Joint Surg Am 1954;36-A(03): 451-475

46 Merle D'Aubigné R. [Numerical classification of the function of the hip. 1970]. Rev Chir Orthop Repar Appar Mot 1990;76(06):371-374

47 Matta JM, Anderson LM, Epstein HC, Hendricks P. Fractures of the acetabulum. A retrospective analysis. Clin Orthop Relat Res 1986; (205):230-240

48 Judet R, Judet J, Letournel E. Fractures of the acetabulum: classification and surgical approaches for open reduction Preliminary report. J Bone Joint Surg Am 1964;46:1615-1646 